\title{
Effect of the fluidic injection on the flow of a converging-diverging conical nozzle
}

\author{
Benderradji Razik $^{1,2^{*}}$, Gouidmi Hamza ${ }^{2,3}$, Beghidja Abdelhadi ${ }^{2}$ \\ ${ }^{I}$ Faculty of Science, Mohamed Boudiaf University, M'sila, ALGERIA \\ ${ }^{2}$ Laboratory of Renewable Energies and Sustainable Development, Universiyu of Constantine 1, ALGERIA \\ ${ }^{3}$ Department of Mechanical Engineering, El-Bachir El-Ibrahimi University of Bordj Bou Arreridj, ALGERIA \\ E-mail*: razik.benderradji@univ-msila.dz
}

\begin{abstract}
The flow in an Over-Expanded Nozzle is subjected to shock waves leading to the unsteady separation of the boundary layer. Free detachment may be followed by a restricted detachment. During the expansion regime in propellant nozzles, several physical phenomena are encountered: supersonic jet, jet separation, adverse pressure gradient, shock wave, turbulent boundary layer, highly compressible mixture layer, return flow, large scale turbulence. These very complex phenomena can considerably affect the performance of the nozzle.The numerical investigation was performed by the CFD-FASTRAN search code, using the $k$-w SST model as the turbulence model. The calculation is performed by solving the Navier-Stokes equations of twodimensional compressible turbulent flow. It is based on the study of the fluidic vectorization phenomenon of the thrust of a double-injection convergent-divergent supersonic conical nozzle. The study is based on the effect of the ratio of NPR pressures with SPR $=1$ on the overall structure of shock waves. The calculation is highlighting the behavior of a flow that has not neglected. In particular, the appearance of the separation zone formed by the fluid jet and the deflection of the main jet cause separation shocks.
\end{abstract}

Keywords: Shock wave, fluidic vectorization, boundary layer, returns flow, Navier-Stokes equations.

Received: 15/05/2020 - Accepted: 21/06/2020

\section{Introduction}

The ancient technique for orienting the jet of a supersonic airplane is the mechanical technique; it is based on all the parts placed at the divergent ax symmetric nozzle. This technique is powerful but expensive. Several problems have marked this mechanical device, especially during take-off or rapid orientation of military flights. So piloting the flight is difficult. It is a question of using the fluid as a solution for orienting the main jet of the supersonic nozzle of the plane. This technique is noted by fluidic vectorization. It is based on the location of a secondary injector at the divergence of the nozzle. In this case, one eliminates all the problems related to the movable fins. However, the fluidic vectorization has some disadvantages mentioned below:
- Delicate installation of injection slots especially in ax symmetric nozzles;

- The shock vectorization method penalizes the thrust coefficient via the total pressure losses through the system of oblique shocks caused by the injection.

The majority of the work, which dealt with the problem of secondary injection of a fluid passing through a main jet [1, 2], who studied the phenomenon of interaction of the gaseous jets in slits crossing supersonic external flows, they showed that the supersonic flow crossing a fluid jet is similar to that when facing a step of a height $h$. Among the researches that have been conducted on this vectorization technique, mention is made in particular of the experimental and numerical work [3-5], numerical and analytical study [6-9]. The stationary steady-state phenomenon of fluidic vectorization in a two-dimensional symmetric supersonic conical convergent-divergent nozzle was studied. 
This study's target is focused on the pressure ratio and overall shock wave structure. We want to emphasize that the action of a flow is not ignored and that the presence of the separation zone created by the fluid jet and the main jet deflection creates shocks in the separation.

\section{Optimization of Numerical Simulation}

The study was conducted on a test case [1]. Basing in this study on the effect of the pressure ratio $\mathrm{NPR}=\mathrm{P}_{\text {io }}$ / $\mathrm{Pa}$, defined by the ratio of the pressure generating the combustion chamber to that of the atmosphere at SPR = $\mathrm{P}_{\text {inj }} / \mathrm{P}_{\text {io }}=1$, which expressed by the ratio of the injection pressure to the pressure generating the combustion chamber. Figure 2 shows the Schematic of split injection pattern distribution and 2D transverse wall pressure. The simulated nozzle is a two-dimensional axisymmetric supersonic conical nozzle, Figure 2, with a section ratio As $/ \mathrm{Ac}=1.8$, and a divergence half-angle of $11.02^{\circ}$. The length of the diverging part is $\mathrm{L}=0.0577 \mathrm{~m}$. The first injector is placed in a fixed position of $\mathrm{x} / \mathrm{xt}=1.8$ and of width $\mathrm{b} 1=0.002032 \mathrm{~m}$, while the second injector of $\mathrm{b} 2=$ $0.001016 \mathrm{~m}$ of width is placed at $\mathrm{x} / \mathrm{xt}=1.4$ of the nozzle neck.

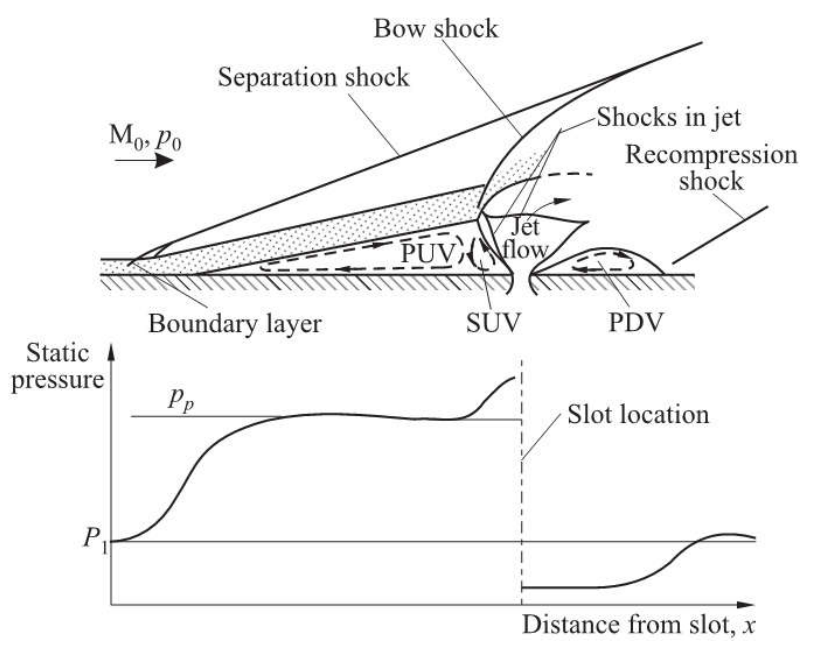

Figure 1. Schematic of split injection pattern distribution and 2D transverse wall pressure [2]

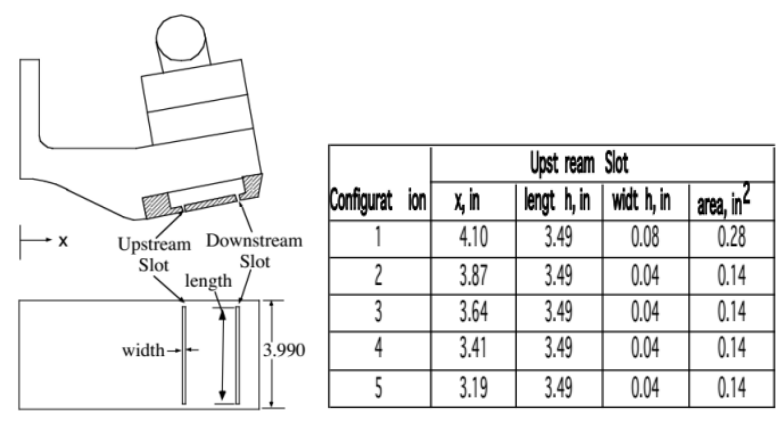

Figure 2. Real photo of NASA nozzle [1]

\section{II.1. CFD-Fastran}

The numerical approach of the CFD-FASTRAN code is based on the resolution of the Navier-Stockes equations by the finite volume method. The flow field is subdivided into small control volumes. Conservation equations are applied to each volume taking into account the flows of variables across each face of the control volume. Each variable is calculated at the center of each cell and assumed to be constant throughout the control volume. The calculation of the convective flux is based on two off-centered diagrams (Upwind). The Roe-FDS scheme (flow difference splitting) and the Van Leer-FVS scheme (vector splitting flow). Spatial precision of order greater than two can be obtained by using a suitable flux limiter. These flux limiters are used to prevent numerical oscillations, in particular for the seat flows of strong interaction zones shocks / discontinuity surfaces. For temporal integration, three schemas are integrated in the code: the explicit Runge-Kutta schema, the semi-implicit schema (implicit point) and the fully implicit schema.

\section{II.2. Mesh and boundary conditions}

Figure 3 shows the profile of the nozzle used in the 2D calculations. The block structured quadrilateral mesh is used. The refinement of the mesh is also taken into account in the vicinity of the walls and that in the wake zones. To better simulate the boundary layers. The domain of calculation comprises 381900 cells. Numerical calculations are performed for turbulent and stationary flow. A subsonic input condition is imposed at the inlet of the nozzle where the generating conditions and the direction of the velocity are imposed. The walls of the nozzle and the upstream outer domains are adherent and adiabatic. The upper and lower boundaries are provided with conditions of non-reflections. Finally, a subsonic exit condition is imposed on the downstream boundary of the domain. This last condition requires a significant longitudinal extension to allow the jet to become subsonic by diffusion of the momentum by the viscosity. The sonic conditions of the gas are applied to the inlet of the injectors are ensured by the laws of isentropic flow related by the ratio of total pressures SPR (according to experience). 


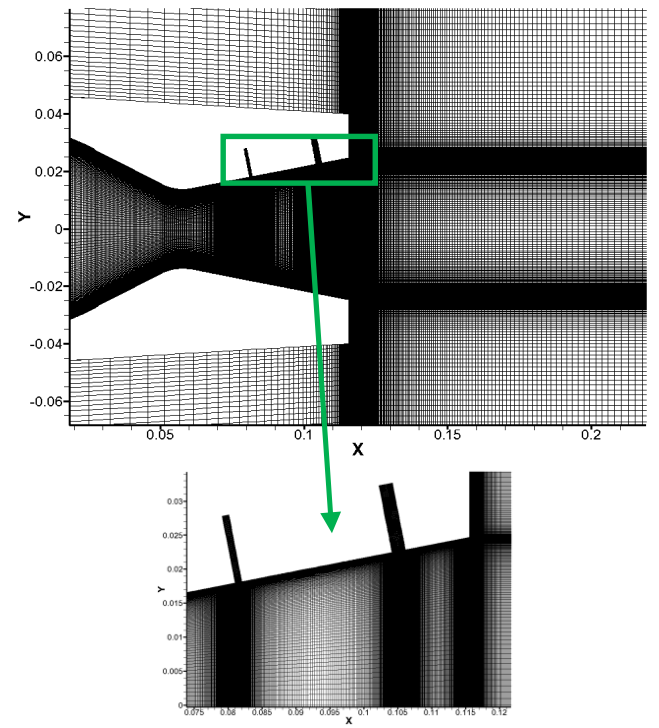

Figure 3. Mesh of the domain of computation

\section{II.3 Influence of Turbulence Models}

In numerical calculations, the choice of the turbulence model significantly affects the results. Several models were tested: the algebraic model of Baldwin_Lomax, the model with an equation of Spalart_Allmaras and the models with two equations of transport $(k-\varepsilon$ and $k-\omega)$. Figure 4 hows the influence of turbulence models on the distribution of wall pressure along the divergent nozzle are injector. The numerical calculations are carried out at NPR $=6$. We notice that all the models used, for example the case of the model k$\varepsilon$. The model of Spalart Allmaras, Balwin Lomax and k$\omega$ reproduces the area of separation appropriately compared to the experiment. Given these results, the k- $\omega$ SST model will be used in the following.

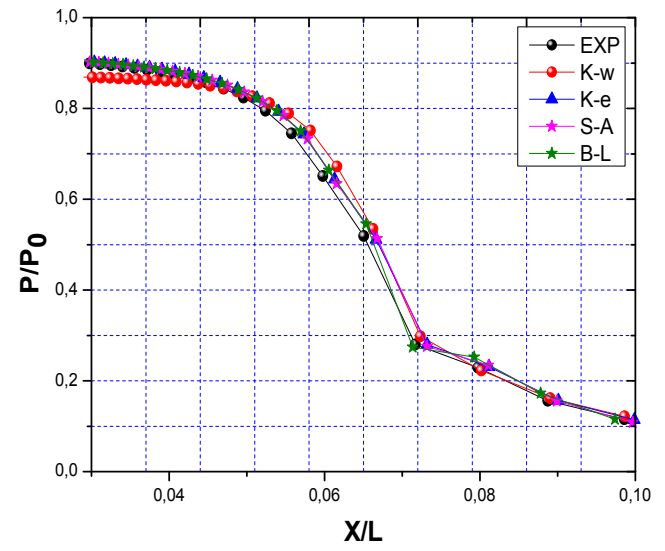

Figure 4. Influence of the turbulence model on the distribution of pressure at NPR $=6$

\section{Validation of results}

Figure 5 represents the partial evolution of pressures and Figure 6 shows the stereoscopy of shock waves. Numerical calculations are compared with the experimental work [1] as shown in Figures 5 and 6. Our results are obtained for NPR $=4.6$ and $\mathrm{SPR}=0.7$. We compared the evolution of parietal pressure between the experiment and our numerical computation by observing that: For the position of the point of separation on both high and low walls. Numeric the point is located at $\mathrm{x} / \mathrm{xt}$ $=1.289$ and at $\mathrm{x} / \mathrm{xt}=1.962$, whereas for the experiment predicts this position at $\mathrm{x} / \mathrm{xt}=1.24$ and at $\mathrm{x} / \mathrm{xt}=1.89$. Also note for the jumps of the pressures, are in agreement between them. A pressure peak can nevertheless be observed on the numerical simulation curve near the inputs of the two injectors, reflecting the presence of the detached shock (Bow Shock). Secondly, we have also compared the experimental stereoscopy with that obtained numerically presented in the form of pressure contours for NPR $=4.6$ and SPR $=0.7$, figure 6 . Two detachment shock waves are observed, one upstream of the injector 2 caused by the enlargement of the nozzle, and another shock wave on the opposite wall near the lip of the nozzle due to the phenomenon of free separation. We can say that the experimental and numerical results are close. So, our numerical results later are validated experimentally.

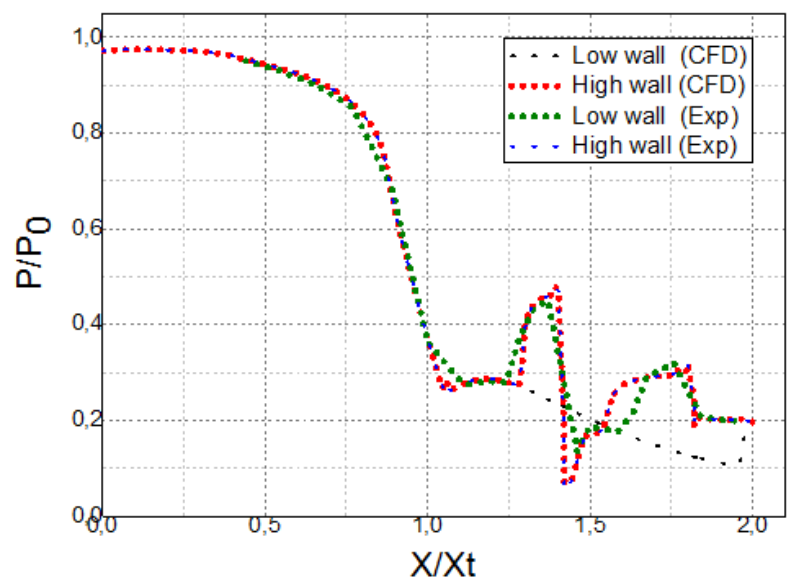

Figure 5. Evolutions of the parietal pressure
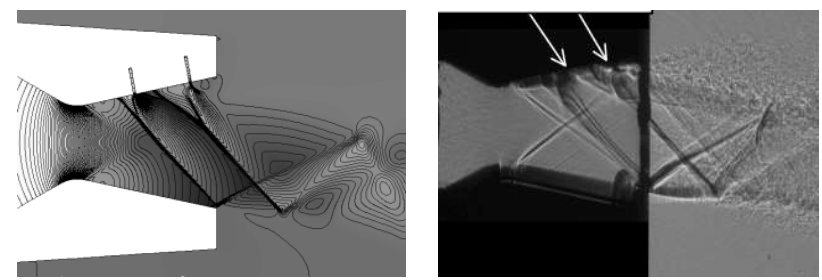

Figure 6. Numerical and experimental stereoscopy, shock waves [1] 


\section{Results and discussion}

We study the effect of the NPR expansion rate on the fluidic vectorization phenomenon in a conical nozzle, with secondary double injection for a stationary compressible turbulent flow. This study deals with the phenomenon of shock interaction as well as the phenomenon of delaminating of the boundary layer in the divergent part of the nozzle. It is carried out for an expansion ratio NPR $=\mathrm{P}_{\mathrm{io}} / \mathrm{P}_{\mathrm{a}}$ ranging from 2 to 8 for an $\mathrm{SPR}=1$, with a position of the injector 2 equal to 1.4 of the throat of the nozzle. Figure 7 shows that the flow regime at the inlet of the two injectors in the divergent nozzle is sonic, corresponds to a Mach number equal to unity $(M=1)$. This value makes it possible to say that this input is assimilated to a sonic neck of a nozzle. This observation is confirmed by the literature [3-5].

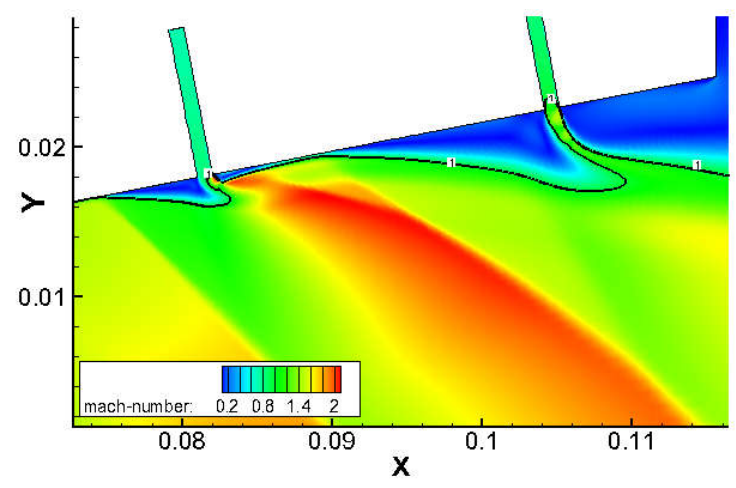

Figure 7. Fields of the Mach number

\section{IV.1 Effect of NPR pressure rate on the overall structure of shock waves}

The effect of the NPR pressure rate on the main jet of the flow is shown in Figure 8 by the iso-contour of the Mach number. For each pressure ratio, the separation obtained is free. It illustrates the stationary aerodynamic field corresponding to the $\mathrm{SPR}=1$ injection ratio. It may also be noted that, regardless of the pressure ratio, the reflection of the detachment shock by the secondary jet with that detached by the isentropic expansion effect on the divergent surface downstream of the neck is regular. This type of reflection has remained to appear for values of the relaxation rate NPR are becomes minimal up to the value of the NPR $=2.5$, where the reflection of Mach is becoming to appear. It can be seen also that the flow at the exit of the nozzle is supersonic followed by a regime of an over-relaxed nozzle. This configuration translated the nature of a flow through a convergent-divergent nozzle. In addition, the size of the recirculation zone that appeared due to the fluidic obstacle is becoming wider with the decrease of the pressure ratio NPR, see Figure 9. This remark is explained at a significant rate of expansion NPR, the main jet is pushed the secondary jet towards the exit of the nozzle, and it decreases its height h. This decrease affects the values of the injected jet heights (fluidic obstacles), $\mathrm{h}_{1}, \mathrm{~h}_{2}$ and $\mathrm{h}_{3}$, on which the lengths and heights of the separation zones $\mathrm{L}_{1}, \mathrm{~L}_{2}, \mathrm{~L}_{3}, \mathrm{H}_{1}$, $\mathrm{H}_{2}$ and $\mathrm{H}_{3}$ depend. These displacements of the shock and the interaction zone are translated by the displacements of the separation points of the boundary layers on the high and low walls. In the opposite direction, when the NPR is weak, the secondary jet is penetrated deeper into the middle of the main jet which has led to a higher jet height. This figure also highlights the recirculation zone associated with detachment and in particular the aspiration of the ambient fluid. There is also a small vortex at the corner of the exit lip of the nozzle in accordance with other numerical results [3-5]. The phenomenon of delaminating, and the interaction between the shock wave / compression shock induced by the boundary layer are presented as follows:

- The first configuration of Mach is captured at NPR = 3.0057 and the second was marked at NPR $=2.5078$, these appearances due to the increase of the angles of the shock waves detached 1 and the detached shock wave due to the second injector, which became strong with the decrease in the rate of NPR pressures. - The total absence of the detached shock wave due to the injector 2 and the Mach reflection labeled with NPR = 2.0208 and 2.5078, respectively. - Beyond NPR $=3.0057$, only observed configuration is the regular reflection, as a remark. The zone of interaction between the detached shock wave and the turbulent boundary layer of the lower wall is increasingly increasing with the decrease of the NPR.

The phenomenon of restricted delaminating is observed for all NPR values.

Jet heights are important when the rate of NPR pressures is decreased. The heights of the separation zones are important when the NPR pressure levels decrease. 

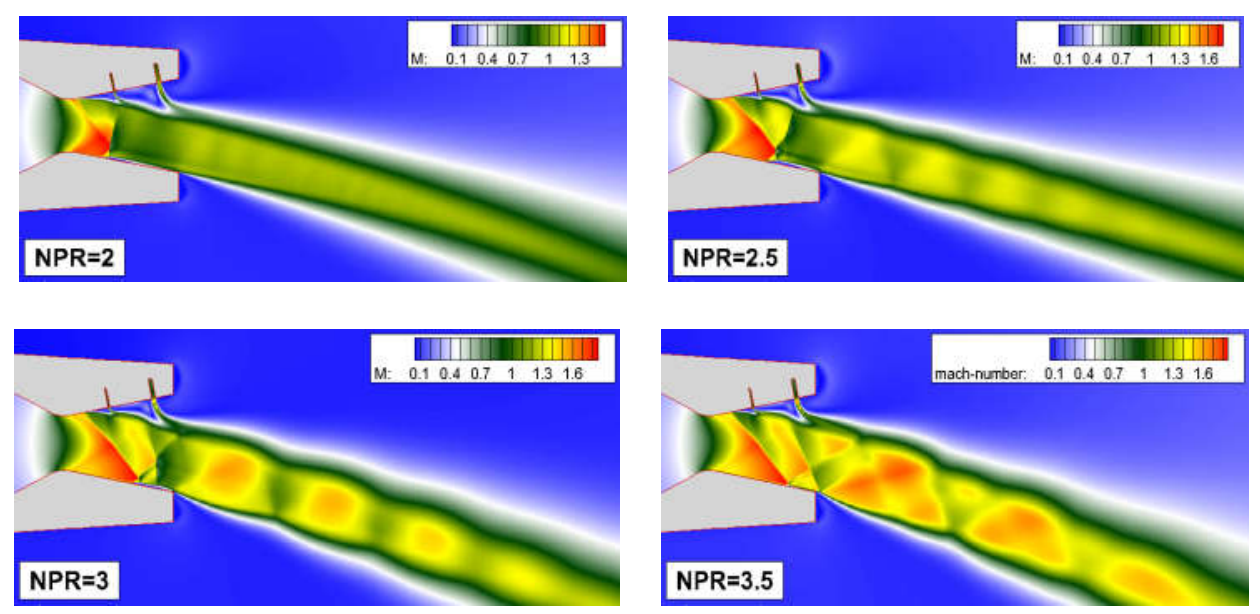

Figure 8. Fields of iso-contours from the Mach number to SPR $=1$ for different NPRs.
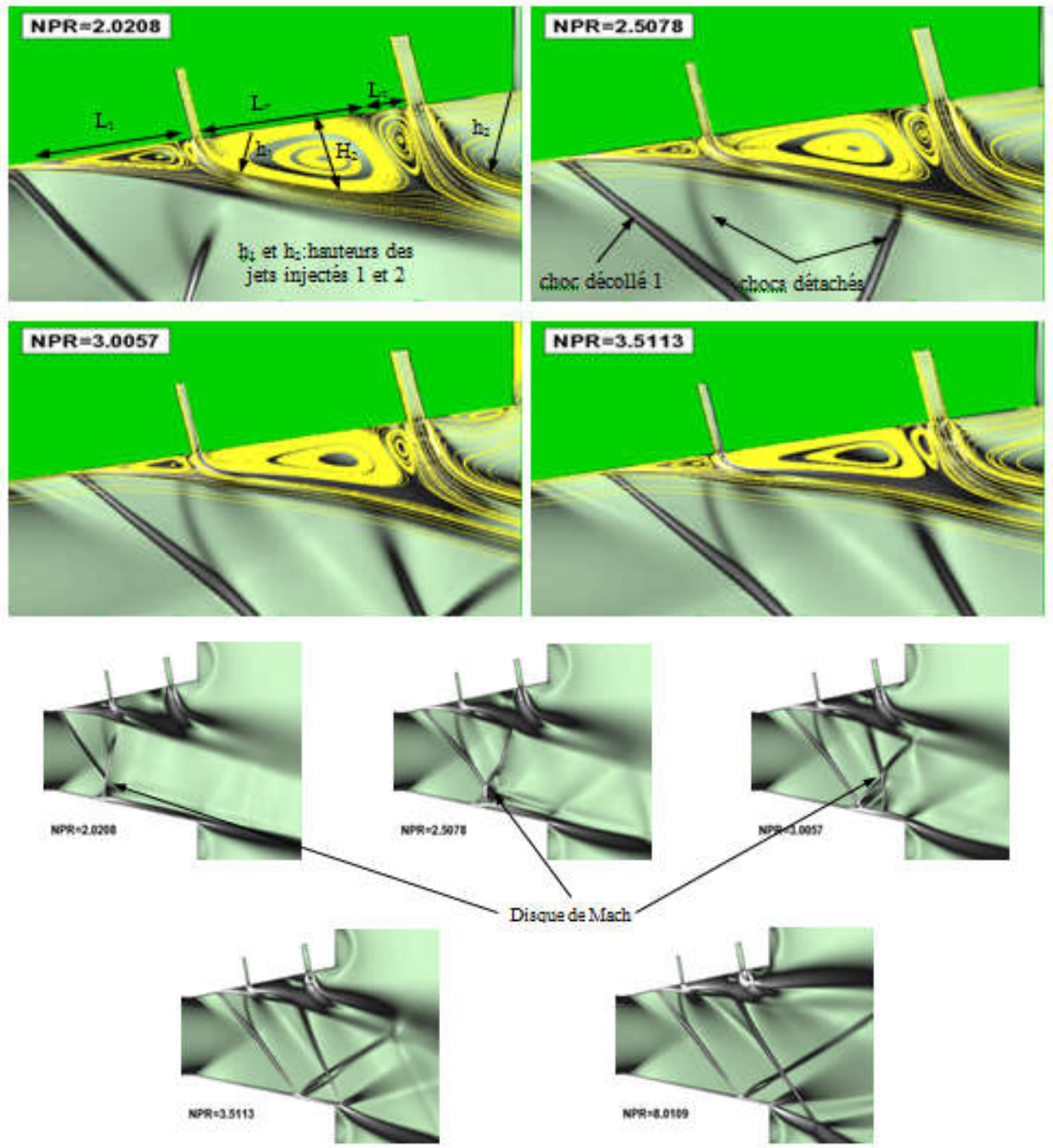

Figure 9. Overall structure of the shock waves and the recirculation zones near the injectors of the upper wall of the nozzle at SPR= 1 , for different NPRs 


\section{IV.2 Variation of the deflection angle of the jet}

Figure 10 shows the deflection angle of the main jet flow versus the NPR expansion ratio for our test case compared with the experiment [1] at $\mathrm{SPR}=0.7$. There is an approach between our value of the deflection angle and that of the experiment. The flow deflection angle as a function of the pressure ratio at $\mathrm{SPR}=1$, gives the same evolutionary trend as that shown in the figure, it is between $8^{\circ}$ and $13.05^{\circ}$. At NPR $=3$, the value of the deflection angle is large, there is a significant deviation of the flow in the direction of the injected jet. When, as the expansion ratio NPR increases, the deflection angle is gradually decreased.

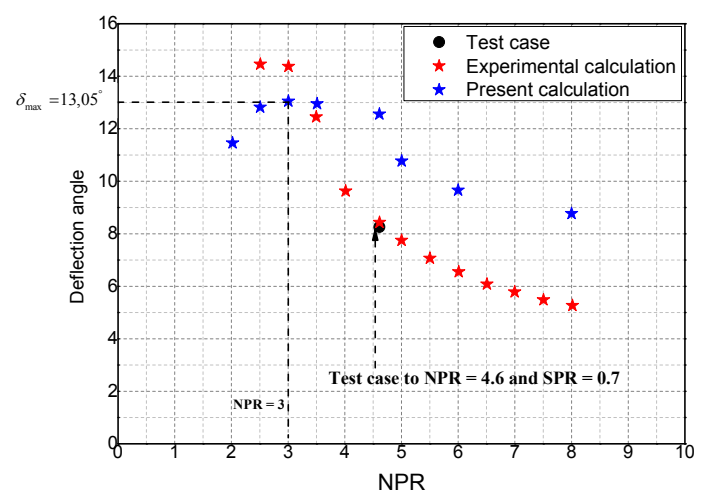

Figure 10. Evolution of the deflection angle of the main jet as a function of the pressure rate NPR at SPR $=1$, for present calculation the width of the jet $2 b_{1}=0.001016 \mathrm{~m}$ and for the calculation of KA Waithe et al [1] $b_{1}=0.002032 \mathrm{~m}$

\section{IV.3 Effects of NPR pressure ratio}

Figures 11 and 12 show the evolution of the parietal pressures as a function of the pressure ratio NPR to $\mathrm{SPR}=1$. It is noted that the separation point and the plateau pressures are well graduated, one on the other following the decrease of the NPR, in particular the value of NPR $=2.0208$, giving a position of the separation point further upstream by compared to others. This shift reflects the recirculation zone which is larger compared to other NPRs cases. This finding is explained by the transition from regular reflection (RR) with small values of NPR to the Mach reflection (MR) that was detected at $\mathrm{NPR}=2.0208$. This transition leads to making the low detachment shock waves weak.

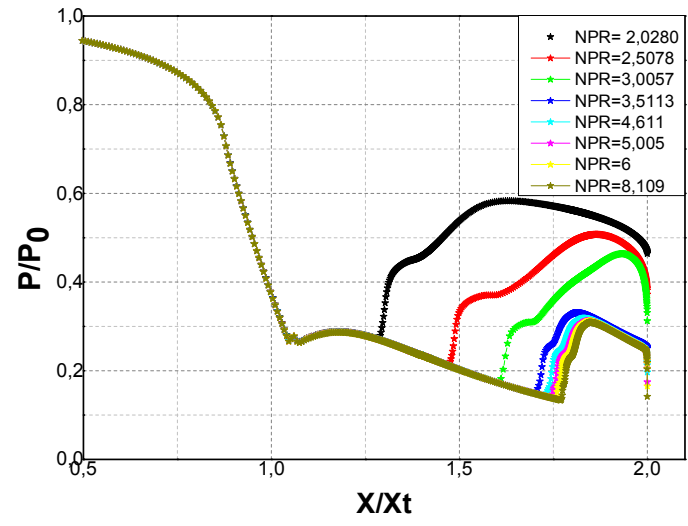

Figure 11. Evolutions of the wall pressure of the upper wall for different $N P R s$ and at $S P R=1$

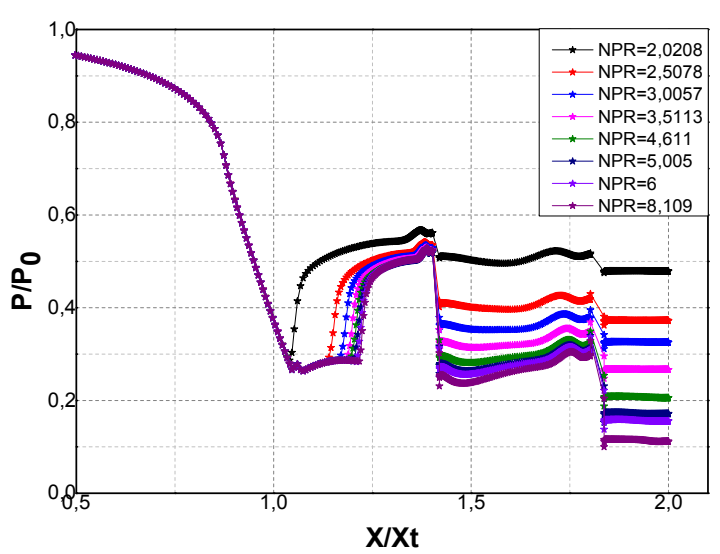

Figure 12. Evolutions of the wall pressure of the lower wall for different NPRs and at SPR $=1$.

\section{Conclusion}

In this study, numerical turbulent twodimensional flow calculations for the investigation of the fluidic vectorization phenomenon of the thrust of a convergent-divergent cone nozzle were presented. These calculations are based on the resolution of stationary Navier-Stokes equations, the CFD-FASTRAN search code, and the k-w SST model as the turbulence model. This code is based on Higher Order's Roe's FDS shockabsorption scheme that is robust to capturing shock. The results obtained are well described this vectorization phenomenon. They conform to those found experimentally and numerically. The transition from regular reflection (RR) with small values of NPR to the Mach reflection (MR) that has been detected induces the existence of a hysteresis phenomenon associated with the shock wave / boundary layer interaction. This type of interaction, it is present in many fields of the aeronautics 
such as: the internal aerodynamic (air intakes, scramjet), external (presence of shock of extrados in transonic, flows in nozzle on-detente). The presence of this interaction in these mechanical systems is a real practical problem because it is at the origin of strong in-stationary constraints, which can lead to the fatigue of structures and their destruction. It can also lead to instabilities in the operation of the engines (pumping of compressors, instability of combustion in scramjet ...).

\section{References}

[1] K. A. Waithe and K. A. Deere, "Experimental and computational investigation of multiple injection ports in a convergent-divergent nozzle for fluidic thrust vectoring", 21st Applied Aerodynamics Conference, June, Orlando, Florida 2003.

[2] F.W. Spaid, E.E. Zukoski, "Study of the interaction of gaseous jets from transverse slotswith supersonic external flows", AIAA J.Vol 6, No 2, 1968, pp. 205-212.

[3] Vladeta Zmijanovic, "Vectorisation fluidique de la poussée d'une tuyère axisymétrique supersonique par injection secondaire", thèse de Doctorat, de l'Université d'Orléans, 2013.

[4] V. Zmijanovic L. Leger, V. Lago, "Experimental and Numerical Study of Thrust Vectoring Effects by Transverse Gas Injection into a Propulsive Axisymmetric C-D Nozzle", AIAA 2012-3874, 48th AIAA/ASME/SAE/ASEE Joint Propulsion Conference \& Exhibit 30 July - 01 August 2012, Atlanta, Georgia.

[5] V. Zmijanovic, V. Lago, L. Leger, E. Depussay, M. Sellam, A. Chpoun, "Thrustvectoring effects of a transverse gas injection into a supersonic cross flow of an axisymmetric convergent-divergent nozzle", progress in Propulsion Physics, Vol. 4, 2013, pp. 227-256.

[6] N. Maarouf, M. Sellam, M. Grignon, and A. Chpoun, "Thrust vectoring through fluidinjection in an axisymmetrical supersonic nozzle: Theoretical and computational study", Journal of shock wave, 2009; pp. 1142- 1146.

[7] A. Ashraf, C. G. Rodriguez, A. J. Neely, and J. Young, "Combination of Fluidic Thrust Modulation and Vectoring in a 2D Nozzle", AIAA 2012-3780, 48thAIAA/ASME/SAE/ASEE Joint Propulsion Conference \& Exhibit, 2012, Atlanta, Georgia.

[8] Xin H. Zou, Qiang Wang, Hagemann, "The Comparative Analysis of Two Typical Fluidic Thrust Vectoring Exhaust Nozzles on Aerodynamic Characteristics", World Academy of Science, Engineering and Technology Vol. 5, 2011, pp. 04-20.

[9] Ahmed Remlaoui, Mohammed Benyoucef, Djamel Assi and Driss Nehari, "A TRNSYS dynamic simulation model for a parabolic trough solar thermal power plant", International Journal of Energetica (IJECA), Vol 4. No 2. 2019. 